\title{
Molecular clouds have power-law PDFs (not log-normal)
}

\author{
João Alves ${ }^{1}$, Marco Lombardi ${ }^{2}$ and Charles Lada ${ }^{3}$ \\ ${ }^{1}$ University of Vienna, Dept.of Astrophysics, Türkenschanzstrasse 17, 1180 Vienna, Austria \\ ${ }^{2}$ University of Milan, Department of Physics, via Celoria 16, I-20133 Milan, Italy \\ ${ }^{3}$ Harvard-Smithsonian Center for Astrophysics, Mail Stop 72, 60 Garden Street, Cambridge, \\ MA 02138, USA
}

\begin{abstract}
Contrary to common belief, the column density PDFs of molecular clouds are not described well by log-normal functions, but are instead power-laws with exponents close to two. We argue that the intrinsic functional form of the PDF cannot be securely determined below $\mathrm{A}_{K} \sim 0.1-0.2 \mathrm{mag}$, limiting our ability to investigate more complex models for the shape of the cloud PDF.
\end{abstract}

Keywords. ISM: clouds, ISM: general, ISM: extinction, ISM: structure

\section{Introduction}

In the recent years, and with the increasing availability of large scale high-dynamic range maps of column density of molecular clouds from dust extinction and emission, the probability distribution function of column density (hereafter PDF) has received much attention. The PDF is arguably one of the easiest quantities to measure. It is predicted by many theoretical studies (e.g. Vazquez-Semadeni 1994; Padoan et al. 1997; Scalo et al. 1998; Federrath et al. 2010) to follow a log-normal distribution, and this prediction has been apparently confirmed (at least up to a few magnitudes of visual extinction) by several observations (Lombardi et al. 2008; Goodman et al. 2009; Lombardi et al. 2010, 2011; Schneider et al. 2013; Alves et al. 2014 but see Tassis et al. 2010). Finally, departures from log-normality at high densities have been associated to the star formation activity of molecular clouds (e.g., Kainulainen et al. 2009; Kainulainen et al. 2014).

\section{PDF completeness is not instrumental completeness}

In Lombardi et al. 2015 we computed the column density PDF for eight nearby complexes using Herschel data and demonstrate that the low end of the PDF is essentially unconstrained by the observations. This is because of projection and boundary bias: the volume probed to derive the PDF is a cone, and intervening or background material along the line of sight essentially makes it impossible to probe the PDFs at low column densities. In other words, molecular cloud boundaries are not well defined in dust emission or extinction maps. Even for clouds relatively distant from the galactic plane (such as Orion, Taurus, or Perseus) it is difficult to reach below $\mathrm{A}_{K} \sim 0.1 \mathrm{mag}$. Our inability to investigate the low end of the PDF severely limits our ability to distinguish its shape, and since $\mathrm{A}_{K} \sim 0.1 \mathrm{mag}$ is, coincidentally we think, near the column density threshold for the $\mathrm{H}_{2}$-to-HI transition, we are forced to conclude that there is no observational evidence for log-normal PDFs in molecular clouds. This statement is restricted to the molecular part of the gas, and does not exclude a possible log-normal PDF for the atomic gas. The observable PDF for Ophiuchus is presented in Figure 1. 


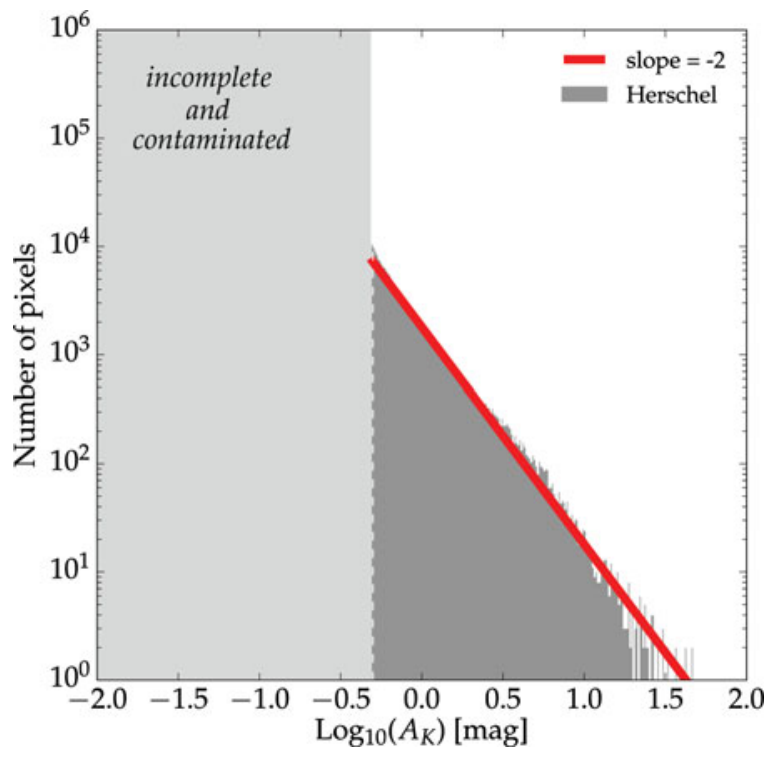

Figure 1. Column density PDF for the Ophiuchus complex from Herschel data (from Alves et al. 2016). Once data completeness is correctly taken into account, both the turn-over and the log-normal like shape at low column densities fall below it, and should be taken as artifacts.

\section{References}

Alves, J., et al. 2014, A\&A, 565, 18

Alves, J., et al. 2016, in prep.

Federrath, C., et al. 2010, A\&A, 512, 81

Goodman, A., et al. 2010, ApJ, 692, 91

Kainulainen, J., et al. 2009, A\& $A, 508$, L35

Kainulainen, J., et al. 2014, Science, 344, 183

Lombardi, M., et al. 2008, A\&A, 489, 143

Lombardi, M., et al. 2010, A\&SA, .512, 67

Lombardi, M., et al. 2011, A\&SA, 535, 16

Lombardi, M., et al. 2015, A\&SA, 576, 1

Padoan, P., et al. 1997, MNRAS, 288, 145

Scalo, J. 1998, ApJ, 288, 145

Schneider, et al. 2013, ApJ, 766, 17

Tassis et al. 2010, MNRAS, 4008, 1089

Vazquez-Semadeni, E. 1994, ApJ, 30, 490 